\title{
Errores sistemáticos en el uso del transportador
}

\section{Systematic errors in the use of the protactor}

\author{
Edinson Fuentes ${ }^{1}$ \\ Martha Leonor Saiz Sáenz² \\ David Fernando Pinzón Piñeros ${ }^{3}$ \\ Universidad Pedagógica y Tecnológica de \\ Colombia
}

\section{RESUMEN}

El error en la adquisición del conocimiento en el aula de clase es una oportunidad de aprendizaje para el estudiante y para el maestro. Es por ello que el docente debe implementar estrategias didácticas que permitan superar los obstáculos que emergen en las interacciones de enseñanza y aprendizaje, no sin antes identificar, clasificar y conocer la naturaleza del error para que éste no se convierta en un conocimiento único que se transfiera a otras disciplinas. En geometría existe una escasa literatura con relación a errores que cometen los estudiantes, por lo que en el presente estudio se planteó como

1 Correo: edinson.fuentes@uptc.edu.co, Teléfono: 3208229561, Universidad Pedagógica y Tecnológica de Colombia, Tunja, ORCID: https://orcid.org/0000-0002-5430$\underline{5016}$

2 Correo: martha.saiz@uptc.edu.co , Teléfono: 3115578936, Universidad Pedagógica y Tecnológica de Colombia, Tunja, ORCID: https://orcid.org/0000-0002-6906$\underline{3953}$

3 Correo: davidfernando001@hotmail.com, Universidad Pedagógica y Tecnológica de Colombia, Tunja, ORCID: https://orcid.org/0000-0003-3538-5775 objetivo general, evaluar información mediante el diseño de una herramienta didáctica que permita recolectar los errores sistemáticos que cometen los estudiantes al usar el transportador de 360 grados como instrumento de medida de la magnitud amplitud angular. El estudio se realizó bajo el paradigma cualitativo, de carácter exploratorio y descriptivo, delimitado por la investigación acción participativa y el marco teórico se sustenta desde los errores en el pensamiento matemático, en geometría y en el uso del instrumento; definición de ángulo como objeto geométrico; construcción matemática del concepto amplitud angular; su magnitud amplitud angular y su medida, y finalmente la medición desde los conceptos asociados al acto de medir y la génesis de la medida en el niño.

PALABRAS CLAVE: error, medición, amplitud angular, transportador. 


\section{ABSTRACT}

The error in the acquisition of knowledge in the classroom is a learning opportunity, for the student and for the teacher, that is why the teacher must implement didactic strategies that allow to overcome the obstacles that emerge in the teaching interactions and learning, but first the nature of the error must be identified, classified and known so that it does not become a unique knowledge that is transferred to other disciplines. In geometry, there is little literature in relation to errors made by students, and therefore, in the present study, the general objective was to evaluate information through the design of a didactic tool that allows collecting the systematic errors that students make when use the $360^{\circ}$ protractor as an instrument to measure the magnitude of the angular amplitude. The study was delimited under the qualitative paradigm, exploratory and descriptive, delimited by participatory action research and the theoretical framework is based on errors in mathematical thinking, geometry and the use of the instrument; definition of angle as geometric object; mathematical construction of the concept of angular amplitude; its magnitude, angular amplitude and its measure, and finally the measurement from the concepts associated with the act of measuring and the genesis of the measure in the child.

KEY WORDS: error, measuring, angular amplitude, protractor.

\section{INTRODUCCIÓN}

El interés por el estudio de la magnitud amplitud angular surge al observar a lo largo de la experiencia docente en los ciclos de educación básica y media, las serias dificultades a las que se enfrentan los estudiantes cuando utilizan el transportador de $360^{\circ}$ al medir amplitudes y al considerar el ángulo como una magnitud. Otro aspecto importante por el cual se considera abordar la temática de estudio se debe a que la revisión de investigaciones publicadas alrededor de este concepto ha sido escasa y los trabajos desarrollados se incluyen en el contexto internacional, pero la gran mayoría de ellos se han enfocado en torno a la comprensión del ángulo como objeto geométrico, en algunos casos a su medida y en pocos casos al instrumento que se utiliza para medir amplitudes en el aula de clase. De esta forma, la constatación de las nulas investigaciones en torno al uso del transportador a nivel local y nacional, y de las serias dificultades que se presentan en el aula de clase que en muchas ocasiones son asociadas al estudiante y no al proceso didáctico o al uso de los instrumentos, se considera interesante llevar a cabo este trabajo que se presenta con el objetivo general de contribuir al análisis de errores sistemáticos que cometen los estudiantes de grado noveno, al usar el transportador de $360^{\circ}$ como instrumento de medida de la magnitud amplitud angular.

La metodología propuesta para la presente investigación es la Investigación - Acción, tomando como enfoque el cualitativo, puesto que permite una interacción constante con los sujetos a investigar y con su propio entorno, de acuerdo con Vasilachis (2006)

el proceso de investigación cualitativa supone: a) la inmersión en la vida cotidiana de la situación seleccionada para el estudio, b) la valoración y el intento por descubrir la perspectiva de los participantes sobre sus propios mundos, y c) la consideración de la investigación como un proceso interactivo entre el investigador y esos participantes, como descriptiva y analítica y que privilegia las palabras de las personas y su comportamiento observable como datos primarios (p. 26).

\section{MARCO TEÓRICO}

El Ministerio de Educación Nacional (MEN) sostiene que, los procesos de enseñanza y aprendizaje de la matemáticas requiere un 
cambio de concepción, concibiéndola como creaciones resultantes de las interacciones y construcciones culturales, como una disciplina en evolución y constante cambio, ello implica una percepción en la formación desde diversos puntos de vista, no solamente, desde el estudiante, sino también desde lo social, lo cultural, lo histórico, pues el reconocimiento y estudio de aspectos que parecen tangentes a la educación, pueden contribuir al reconocimiento de las dificultades que se presentan en el aula de clase MEN (2003).

De acuerdo con Abrate et al. (2006), los cimientos del conocimiento matemático son abstracción reflexiva, donde los errores se encuentran inmersos en el desarrollo y avance del conocimiento científico. Por tanto, se debe reconocer la importancia de identificar y caracterizar los errores de los estudiantes en los procesos de aprendizaje, y concretar las acciones y estrategias de enseñanza, de tal manera, que se pueda abordar el error de forma positiva.

En este orden de ideas, una oportunidad surge cuando se tiene la identificación del error, este le permitirá al maestro, obtener información respecto al origen, a la frecuencia con la que ocurre y al diseño de actividades de planeación que permitan superar el mismo. Los errores han sido objeto de diversas investigaciones en los procesos de enseñanza y aprendizaje, en el área de las matemáticas se destacan Brousseau et al. (1986); Rico (1995); Astolfi (1999); Pochulu (2005).

En estas investigaciones sobresale el papel que tiene el error en la enseñanza y aprendizaje de las matemáticas y se reflexiona en torno a esa expresión exteriorizada de la comprensión cognitiva de una persona, en el que están inmersas variables como: el rol del docente, rol del estudiante, el currículo, contexto sociocultural, recursos utilizados, entre otras. Además, los autores consideran que los errores no son punitivos ni fallos exclusivos del estudiante: debido a que es una actividad que se encuentra inmersa en la adquisición del conocimiento y también intervienen el entorno, los saberes previos y los docentes, además, que permiten señalar los obstáculos que desafía el estudiante en el aprendizaje de la matemática.

Los errores en el contexto educativo son parte importante en el desarrollo del campo científico a nivel general. De hecho, el avance de estos se da por el reconocimiento, análisis y posterior superación, así, no solamente se presentan en el desarrollo del cálculo, también se pueden encontrar en el aprendizaje de la geometría, de acuerdo con Cantoral et al. (2005), los estudiantes presentan serias dificultades al enfrentarse a los cursos de geometría, lo que implica en un primer momento un conocimiento del error para motivar estrategias didácticas para su prevención.

Al respecto, Franchi y Hernández (2004), proponen una tipología que permite identificar y clasificar los errores de los alumnos en el área de geometría, subrayan los siguientes: errores del lenguaje geométrico, de gráficos, de razonamiento, de transferencia, de técnica, de tecnología y azarosos.

Así mismo, Chamorro (1995), hace referencia a la dificultad que presentan los estudiantes cuando miden una longitud, pues el acto de medir implica reconocer y separar la longitud del objeto de otras cualidades, asimilar la longitud real con un objeto como un segmento de recta, reconocer que el origen de la graduación del instrumento es el cero, además de hacer coincidir este origen correctamente con el objeto a medir, lo dicho hasta aquí supone que, los estudiantes reconocen algunos conceptos tradicionales, pero se les dificulta reconocer los técnicos, lo cual genera una inapropiada compresión y confusión con el objeto de estudio.

Se espera que finalizando octavo y noveno grado 
el estudiante debe estar en la capacidad de utilizar un transportador para medir la amplitud angular, sin embargo, autores como Rotaeche y Montiel (2017), sostienen que estudiantes de secundaria, muestran dificultades, así como, conflictos y malentendidos que son evidencia de fenómeno didáctico asociado a la particularidad del concepto ángulo, y estas se presentan independientemente de la población, contexto o enfoque de enseñanza.

De acuerdo con Rotaeche y Montiel (2017), un conflicto frecuente se presenta cuando los estudiantes usan el transportador al medir ángulos en una circunferencia, debido a que una de las dificultades es usarlo como regla para medir la amplitud angular. Es importante resaltar, que, en el proceso de enseñanza y aprendizaje, se presta poca atención a la diferenciación entre el procedimiento empleado y el instrumento de medición que se utiliza para obtener la medida de un ángulo. Por ejemplo, Casas y Luengo (2005), Mitchelmore y White (1998), destacan las dificultades explícitas en la medición de la amplitud angular, afirman que los estudiantes al medir la amplitud de ángulos asumen que la longitud de las rectas que definen el ángulo afecta su medida, así como, en la dificultad de reconocer la amplitud angular de medidas como $0^{\circ}, 180^{\circ}$ y $360^{\circ}$.

Estas dificultades no son ajenas, a los estudiantes de grado noveno del Colegio Próspero Pinzón, la asignatura de geometría se encuentra ubicada desde grado primero a grado once y aborda el estudio de la geometría, tanto métrica como a nivel analítico del plano. Esta es una asignatura de carácter formativo, la cual potencia el uso del razonamiento formal y sistemático para la resolución de problemas. Desde la experiencia se evidencia que el estudiante presenta mayores dificultades para aprender la geometría métrica, lo que podría asociarse a la exigencia del razonamiento lógico-formal. Los docentes dedican gran parte del tiempo para hacer énfasis en los puntos álgidos del contenido.

En esta línea de errores sistemáticos, diferentes autores como Rico (1995) han propuesto modelos para clasificarlos, y entender su procedencia. Atendiendo a los objetivos del presente estudio, es necesario considerar esta tipología de errores, para la consolidación de las categorías de análisis.

\section{MODELO DE CLASIFICACIÓN DE ERRORES}

A continuación, se relacionan diferentes autores como Astolfi (1999) (Tabla 1), Movshovitz et al. (1987) (Tabla 2), Raddatz (1979) (Tabla 3), Socas (1997) (Tabla 4), Franchi y Hernández (2004) (Tabla 5), quienes muestran algunas formas de clasificar los errores y la manera como el estudiante los aborda.

Astolfi (1999), afirma que "los errores se presentan como síntomas principales dentro del proceso de enseñanza y aprendizaje logrando con esto identificar las dificultades de los estudiantes" (p.49). Además, sostiene que el error debe ser el centro del proceso de enseñanza aprendizaje, para poder realizar una mejor intervención educativa.

De acuerdo con los planteamientos, el autor exterioriza diferentes modelos que conllevan a la clasificación del error, señalando que el estudiante puede cometer el mismo en cualquier situación o asignatura, en particular, cuando se trata de matemática los estudiantes suelen ejecutar errores como: 
Tabla 1. Modelo de clasificación de errores de Astolfi.

\begin{tabular}{|c|c|}
\hline Errores & Procedencia \\
\hline $\begin{array}{l}\text { De redacción y } \\
\text { comprensión de las } \\
\text { instrucciones de trabajo }\end{array}$ & $\begin{array}{l}\text { Estos son errores que emergen de dificultades asociadas al } \\
\text { entendimiento y comprensión de la verbalización de los enunciados de } \\
\text { los problemas y situaciones propuestas }\end{array}$ \\
\hline $\begin{array}{l}\text { Resultan de } \\
\text { una inadecuada } \\
\text { interpretación }\end{array}$ & $\begin{array}{l}\text { Los estudiantes no utilizan los conocimientos previos para resolver } \\
\text { situaciones problémicas nuevas, no utilizan su razonamiento para } \\
\text { resolver problemas, responde de acuerdo con las exigencias que creen el } \\
\text { profesor solicita. }\end{array}$ \\
\hline $\begin{array}{l}\text { Del resultado de } \\
\text { las concepciones } \\
\text { alternativas de los } \\
\text { estudiantes }\end{array}$ & $\begin{array}{l}\text { Procede de conocimientos y procedimientos consolidados que en } \\
\text { algunas situaciones produjeron respuestas exitosas, y al adaptarlos a una } \\
\text { nueva situación resultan en equivocación. }\end{array}$ \\
\hline $\begin{array}{l}\text { Ligados a las } \\
\text { operaciones } \\
\text { intelectuales implicadas }\end{array}$ & $\begin{array}{l}\text { Algunas veces las redes conceptuales que el alumno debe activar } \\
\text { para abordar alguna situación no se encuentran consolidadas debido a } \\
\text { capacidades lógico-matemáticas distintas. }\end{array}$ \\
\hline $\begin{array}{l}\text { Debido a una sobrecarga } \\
\text { cognitiva de la actividad } \\
\text { a realizar }\end{array}$ & $\begin{array}{l}\text { No se dispones de tiempo para reflexionar, buscar información, inferir, } \\
\text { etc. Se exige en la resolución del problema, la memoria del estudiante y } \\
\text { la resolución inmediata. }\end{array}$ \\
\hline $\begin{array}{l}\text { Con origen en otra } \\
\text { disciplina }\end{array}$ & $\begin{array}{l}\text { Procede de ignorar contenidos de otros campos de conocimiento } \\
\text { necesarios para abordar y dar respuesta a la situación propuesta. }\end{array}$ \\
\hline $\begin{array}{l}\text { Causados por la } \\
\text { complejidad propia del } \\
\text { contenido }\end{array}$ & $\begin{array}{l}\text { Procede de la situación y el esfuerzo cognitivo que demanda el abordarla } \\
\text { y que varía dependiendo de su complejidad. }\end{array}$ \\
\hline
\end{tabular}

Fuente: elaboración propia, adaptada de Astolfi (1999).

Tabla 2. Modelo de clasificación de errores de Movshovitz et al.

\begin{tabular}{l|l}
\hline \multicolumn{1}{c|}{ Errores } & \multicolumn{1}{c}{ Procedencia } \\
\hline $\begin{array}{l}\text { Debidos a datos utilizados } \\
\text { inadecuadamente }\end{array}$ & $\begin{array}{l}\text { Proceden la forma como el estudiante utiliza y relaciona } \\
\text { inadecuadamente los datos para aplicarlos en la solución de } \\
\text { problemas. }\end{array}$ \\
\hline $\begin{array}{l}\text { Debidos a incorrecta } \\
\text { interpretación del lenguaje }\end{array}$ & $\begin{array}{l}\text { Proceden de incorrectas interpretaciones entre aspectos } \\
\text { matemáticos y el lenguaje coloquial. }\end{array}$ \\
\hline $\begin{array}{l}\text { Debidos a inferencias } \\
\text { lógicamente invalidas }\end{array}$ & $\begin{array}{l}\text { Surgen por un razonamiento incorrecto. que luego, el estudiante } \\
\text { utiliza para resolver el problema planteado. }\end{array}$ \\
\hline $\begin{array}{l}\text { Debidos al uso de teoremas } \\
\text { o definiciones deformadas }\end{array}$ & $\begin{array}{l}\text { Surgen por una deformación de fundamentos matemáticos como, } \\
\text { reglas, postulados, teoremas o definiciones. }\end{array}$ \\
\hline $\begin{array}{l}\text { Debidos a ausencia de } \\
\text { retrospección o proceso de } \\
\text { verificación de la solución }\end{array}$ & $\begin{array}{l}\text { Surgen porque el estudiante no realiza una verificación de la } \\
\text { solución, de ser así, hubiera eliminado el error. }\end{array}$ \\
\hline
\end{tabular}




\begin{tabular}{l|l}
\hline Técnicos & $\begin{array}{l}\text { Se contemplan en esta categoría lo errores producto de } \\
\text { manipulación de expresiones, cálculos y obtención de datos de } \\
\text { tablas o gráficas, entre otros. }\end{array}$ \\
\hline
\end{tabular}

Fuente: Elaboración propia, adaptada de Movshovitz et al. (1987).

Tabla 3. Modelo de clasificación de errores de Raddatz.

\begin{tabular}{l|l}
\hline \multicolumn{1}{c|}{ Errores } & \multicolumn{1}{c}{ Procedencia } \\
\hline $\begin{array}{l}\text { Debidos a la dificultad del } \\
\text { lenguaje }\end{array}$ & $\begin{array}{l}\text { Los alumnos consideran que el estudio de las matemáticas tiene } \\
\text { una dificultad adicional en relación con el lenguaje de símbolos, y } \\
\text { conceptos propios de la matemática. }\end{array}$ \\
\hline $\begin{array}{l}\text { Debidos a dificultades para } \\
\text { representar información y } \\
\text { relaciones espaciales }\end{array}$ & $\begin{array}{l}\text { Proceden de la dificultad para establecer relaciones y } \\
\text { representaciones espaciales requeridas para abordar y solucionar } \\
\text { problemas matemáticos o situaciones geométricas. }\end{array}$ \\
\hline $\begin{array}{l}\text { Debidos a un aprendizaje } \\
\text { deficiente de hechos, } \\
\text { destrezas y conceptos } \\
\text { previos }\end{array}$ & $\begin{array}{l}\text { Surgen de saberes deficientes o ausentes en relación con algoritmos } \\
\text { conceptos entre otros. }\end{array}$ \\
\hline $\begin{array}{l}\text { Debidos a la rigidez de } \\
\text { pensamiento }\end{array}$ & $\begin{array}{l}\text { Proceden de la rigidez del pensamiento y la dificultad de adaptar } \\
\text { saberes a nuevas situaciones, aquí se contemplan errores de } \\
\text { adaptación, asociación y aplicación de fundamentos matemáticos. }\end{array}$ \\
\hline $\begin{array}{l}\text { Debidos a la aplicación } \\
\text { de reglas o estrategias } \\
\text { irrelevantes }\end{array}$ & $\begin{array}{l}\text { Proceden de aplicación de algoritmos incorrectos y ausencia de } \\
\text { estrategias para solucionar situaciones matemáticas. }\end{array}$ \\
\hline
\end{tabular}

Fuente: elaboración propia, adaptada de Raddatz (1979), citado en Franchi y Hernández (2004).

Tabla 4. Modelo de clasificación de errores de Socas.

\begin{tabular}{l|l}
\hline \multicolumn{1}{c|}{ Errores } & \multicolumn{1}{c}{ Procedencia } \\
\hline $\begin{array}{l}\text { Que tienen su origen en un } \\
\text { obstáculo }\end{array}$ & $\begin{array}{l}\text { Se concibe los obstáculos como saberes consolidados } \\
\text { adecuadamente, no como ausencia de conocimiento. }\end{array}$ \\
\hline $\begin{array}{l}\text { Que tienen su origen en la } \\
\text { ausencia de sentido: Estos } \\
\text { pueden dividirse en tres } \\
\text { clases }\end{array}$ & $\begin{array}{l}\text { Existen nueve errores asociados a procedimientos incorrectos como } \\
\text { manipulación de fórmulas y aplicaciones de reglas; también existen } \\
\text { nueve errores producido de ausencia de asimilación de relaciones } \\
\text { y procedimientos aritméticos, y nueve errores procedentes de } \\
\text { interpretaciones inadecuadas del lenguaje propio de la matemática. }\end{array}$ \\
\hline $\begin{array}{l}\text { Que tienen su origen } \\
\text { en actitudes afectivas y } \\
\text { emocionales }\end{array}$ & Proceden de dificultades de concentración. \\
\hline
\end{tabular}

Fuente: elaboración propia, adaptada de Socas (1997). 
Por último, Franchi y Hernández (2004), proponen una tipología de errores que surge a partir del estudio de la clasificación de errores de Raddatz (1979), Astolfi (1999), Movshovitz et al. (1987) y Socas (1997), señalaron unos errores que no se clasificaron porque no encontraron categorías específicas en el área de la geometría, de esta manera, a diferencia de las anteriores clasificaciones, los autores focalizaron su estudio a proponer una tipología de errores particular para el campo de la geometría plana.

Tabla 5. Modelo de clasificación de errores de Franchi y Hernández.

\begin{tabular}{l|l}
\hline \multicolumn{1}{c|}{ Errores } & \multicolumn{1}{c}{ Procedencia } \\
\hline De Pre-requisitos & $\begin{array}{l}\text { Procede de la deficiencia en saberes, hechos, aprendizajes y } \\
\text { habilidades que debieron haber sido apropiados por el estudiante previo } \\
\text { al trabajo y desarrollo de saberes de la geometría. }\end{array}$ \\
\hline Propios del lenguaje & $\begin{array}{l}\text { Provienen de dificultades en la interpretación del lenguaje y sistema } \\
\text { de símbolos propios de la geometría y posiblemente se relaciona con } \\
\text { conflictos del lenguaje cotidiano. }\end{array}$ \\
\hline Gráficos & $\begin{array}{l}\text { Procede de dificultades con figuras geométricas en situaciones de } \\
\text { interpretación, representación e imaginación. }\end{array}$ \\
\hline De razonamiento & $\begin{array}{l}\text { Procede de la dificultad para usar adecuadamente las equivalencias e } \\
\text { implicaciones lógicas, definiciones, teoremas, axiomas y corolarios. }\end{array}$ \\
\hline De transferencia & $\begin{array}{l}\text { Procede de la dificultad de extrapolar saberes de otros campos del } \\
\text { conocimiento útiles para abordar situaciones reales. }\end{array}$ \\
\hline De técnica & $\begin{array}{l}\text { Procede de dificultades para aplicar correctamente algoritmos o } \\
\text { procedimientos de demostración en situaciones donde se abordan } \\
\text { proposiciones geométricas. }\end{array}$ \\
\hline De tecnología & $\begin{array}{l}\text { Procede de escoger inadecuadamente algoritmos o estrategias para } \\
\text { desarrollar demostraciones en geométrica. }\end{array}$ \\
\hline Azarosos & $\begin{array}{l}\text { Procede de manipulación, sustitución o remplazamiento de símbolos } \\
\text { inadecuadamente; aquí se incluyen expresiones algebraicas y } \\
\text { aritméticas. }\end{array}$ \\
\hline
\end{tabular}

Fuente: elaboración propia, adaptada de Franchi y Hernández (2004). 
A continuación, se enunciarán algunos autores que determinan errores en torno al uso del instrumento de medida longitud y a tareas conceptualmente orientadas, los cuales serán adaptados a los errores que los estudiantes cometen cuando usan el transportador como medida de la amplitud angular.
Frías et al. (2001), propone algunos errores alrededor del uso de instrumentos que permiten determinar la cantidad de magnitud longitud (Tabla 6).

Tabla 6. Errores en el uso del instrumento de Frías et al.

\section{Errores}

No hacer coincidir o alinear el cero del instrumento con uno de los extremos de la longitud que se está midiendo, este error puede estar inducido si el instrumento no inicia en cero.

Contar las marcas del instrumento como la cantidad de unidades del segmento a medir. Por lo tanto, la medida resultante es mayor en una unidad.

Empezar la medición en una marca distinta de cero y no se resta a la medida obtenida el punto en el que se ubicó inicialmente.

Considerar que no se puede asignar una cantidad de medida a una línea curva.

Fuente: elaboración propia, adaptada de Frías et al. (2001).

En el mismo sentido, Sisman y Aksu (2016) establecen los siguientes errores:

Tabla 7. Errores en el uso del instrumento Sisman y Aksu.

\begin{tabular}{l}
\hline \multicolumn{1}{c}{ Errores } \\
\hline Iniciar la secuencia numérica en uno en vez de iniciar en cero. \\
\hline El instrumento de medida debe tener una cantidad de magnitud mayor que la del objeto que se está \\
midiendo. \\
\hline Error asociado a la aditividad al concepto de iteración de una unidad y a los elementos estructurales \\
de una regla. \\
\hline No considera la conversión de unidades al medir en centímetros. \\
\hline No considera la conversión de unidades al medir en centímetros. \\
\hline Si el instrumento no tiene la medida cero, no sirve para medir.
\end{tabular}

Fuente: elaboración propia, adaptada de Sisman y Aksu (2016).

Investigaciones como las de Mitchelmore y White (1998), Casas y Luengo (2005), Rotaeche y Montiel (2009), muestran los conflictos de los estudiantes en la compresión y medición de ángulos (Tabla 8), los cuales están asociados a la noción conceptual del objeto de estudio $y$ al instrumento que se utiliza para realizar la metrización en la clase: el transportador. 
Tabla 8. Errores en la medición de ángulos de Mitchelmore y White; Casas y Luengo; Rotaeche y Montiel.

\section{Errores}

Conceptualizar el Ángulo desde la medida de la amplitud angular como giro e inclinación.

Reconocer en figuras bidimensionales los ángulos que son delimitados por los lados del polígono. Identificar los ángulos cuya amplitud corresponde a la cantidad de $0^{\circ}, 180^{\circ}$ y $360^{\circ}$.

Considerar que la cantidad de medida de magnitud de un ángulo es afectada por la longitud de los rayos o lados que componen el objeto.

Usar el transportador como regla para medir la amplitud angular.

Fuente: Elaboración propia, adaptada de errores en la medición de ángulos de Mitchelmore y White (1998), Casas y Luengo (2005), Rotaeche y Montiel (2009).

Antes de abordar las consideraciones didácticas de los saberes que permiten comprender las dificultades que se reflejan en los errores que cometen los estudiantes cuando usan el transportador para medir la amplitud de ángulos, se requiere en primera instancia comprender y considerar las configuraciones conceptuales desde la matemática y la geometría de los objeto de estudio, por esta razón, a continuación se presentan la definición de ángulo desde la geometría, y la construcción de la magnitud amplitud angular, desde la matemática formal.

\section{DEFINICIÓN DE ÁNGULO COMO OBJETO GEOMÉTRICO}

Los babilonios calcularon la medida de los ángulos en grados, minutos y segundos. Al igual que los egipcios $y$ los griegos, los babilonios descubrieron que podían estimar su posición en la tierra con la de las estrellas midiendo la altura de estas con respecto al horizonte, por lo que se podría afirmar que la Geometría y la Astronomía fueron dos ciencias que nacieron simultáneamente (Guzmán, 2014, p.10).

Por otro lado, Euclides propone en sus Elementos:

Definición 8: Un ángulo plano es la inclinación entre dos líneas en un plano, que se unen la una a la otra y que no están sobre una línea recta.

Definición 9: $Y$, cuando las líneas que contienen al ángulo son rectas, el ángulo se llama rectilíneo.

De acuerdo con Casas y Luengo (2005), ya desde los Elementos de Euclides, se presenta una definición imprecisa del concepto ángulo, pues la primera definición, muestra una concepción de ángulo como la "inclinación de dos líneas" entre dos rectas, en la segunda se define como algo contenido "entre dos líneas". Ambas definiciones encierran, excluyen a los ángulos de $0^{\circ}$ y $180^{\circ}$, como la exclusión o la posibilidad de que los lados del ángulo puedan ser líneas curvas.

\section{ANÁLISIS DE RESULTADOS Y DISCUSIÓN}

Para recopilar la información de cada una de las actividades, se seleccionaron los resultados de los estudiantes que hicieron todas las actividades, lo cual permitió evidenciar el comportamiento frente a las categorías de análisis y las subcategorías formuladas. Se asumió el trabajo de cada estudiante codificándolos desde (E1 a E34), la asignación de la subcategoría se realiza de la siguiente manera: cero $(0)$ indica que el estudiante NO comete el error de la subcategoría propuesta, 
uno (1) indica que el estudiante SI comete el error de la subcategoría.

\section{ACTIVIDAD 1 (A1)}

El siguiente gráfico ilustra un transportador, coloca los números como deben ir en el transportador.

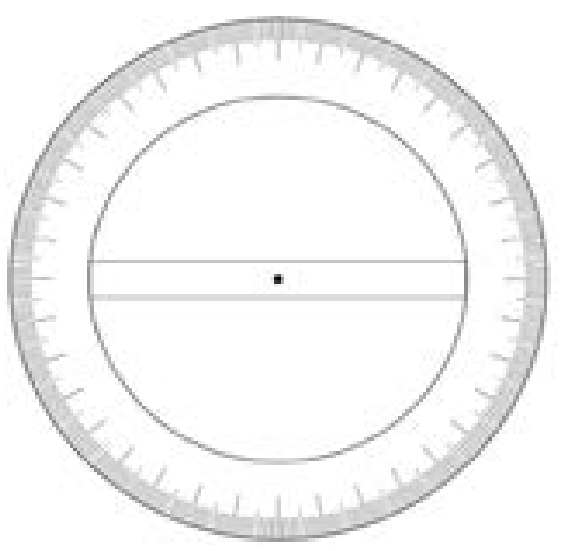

Figura 1. ilustración de transportador. Esta figura, se encuentra a escala 1:1 de la original. Elaboración propia.

Tabla 9. Información recolectada y clasificada actividad 1 (A1).

\begin{tabular}{|c|c|c|c|c|c|c|c|c|c|c|c|}
\hline \multirow{2}{*}{ Estudiante } & \multicolumn{9}{|c|}{ Categoría 1 } & \multicolumn{7}{|c|}{ Categoría 2 } \\
\cline { 2 - 14 } & & & & & & & & & & & \\
\hline E1 & 0 & 0 & 0 & 0 & 0 & 0 & 0 & 0 & 0 & 0 & 0 \\
\hline E2 & 1 & 0 & 1 & 0 & 1 & 0 & 0 & 0 & 0 & 0 & 0 \\
\hline E3 & 0 & 0 & 0 & 0 & 0 & 0 & 0 & 0 & 0 & 0 & 0 \\
\hline E4 & 0 & 0 & 0 & 0 & 0 & 0 & 0 & 0 & 0 & 0 & 0 \\
\hline E5 & 0 & 0 & 0 & 0 & 0 & 0 & 0 & 0 & 0 & 0 & 0 \\
\hline E6 & 0 & 0 & 0 & 0 & 0 & 0 & 0 & 0 & 1 & 1 & 0 \\
\hline E7 & 0 & 0 & 0 & 0 & 0 & 0 & 0 & 0 & 1 & 1 & 0 \\
\hline E8 & 0 & 0 & 0 & 0 & 0 & 0 & 0 & 0 & 0 & 0 & 0 \\
\hline E9 & 0 & 0 & 0 & 0 & 0 & 0 & 0 & 0 & 0 & 0 & 0 \\
\hline E10 & 1 & 0 & 1 & 0 & 1 & 0 & 0 & 0 & 0 & 0 & 0 \\
\hline E11 & 0 & 0 & 0 & 0 & 0 & 0 & 0 & 0 & 0 & 0 & 0 \\
\hline E12 & 0 & 0 & 0 & 0 & 0 & 0 & 0 & 0 & 0 & 0 & 0 \\
\hline E13 & 0 & 0 & 0 & 0 & 0 & 0 & 0 & 0 & 0 & 0 & 0 \\
\hline E14 & 0 & 0 & 0 & 0 & 0 & 0 & 0 & 0 & 0 & 0 & 0 \\
\hline E15 & 0 & 0 & 0 & 0 & 0 & 0 & 0 & 0 & 0 & 0 & 0 \\
\hline E16 & 0 & 0 & 0 & 0 & 0 & 0 & 0 & 0 & 0 & 0 & 0 \\
\hline E17 & 0 & 0 & 0 & 0 & 0 & 1 & 0 & 1 & 0 & 0 & 0 \\
\hline E18 & 0 & 1 & 0 & 0 & 0 & 1 & 1 & 0 & 0 & 0 & 0 \\
\hline E19 & 0 & 0 & 0 & 0 & 0 & 0 & 0 & 0 & 0 & 0 & 0 \\
\hline
\end{tabular}




\begin{tabular}{|c|c|c|c|c|c|c|c|c|c|c|c|}
\hline E20 & 0 & 0 & 0 & 0 & 0 & 0 & 0 & 0 & 0 & 0 & 0 \\
\hline E21 & 0 & 0 & 0 & 0 & 0 & 1 & 1 & 0 & 0 & 0 & 0 \\
\hline E22 & 0 & 0 & 0 & 0 & 0 & 0 & 0 & 0 & 0 & 0 & 0 \\
\hline E23 & 0 & 0 & 0 & 0 & 0 & 0 & 0 & 0 & 0 & 0 & 0 \\
\hline E24 & 0 & 0 & 0 & 0 & 0 & 0 & 0 & 0 & 0 & 0 & 0 \\
\hline E25 & 0 & 0 & 0 & 0 & 0 & 0 & 0 & 0 & 0 & 0 & 0 \\
\hline E26 & 0 & 0 & 1 & 0 & 0 & 0 & 0 & 0 & 1 & 1 & 0 \\
\hline E27 & 0 & 0 & 0 & 0 & 0 & 0 & 0 & 0 & 0 & 0 & 0 \\
\hline E28 & 0 & 0 & 0 & 0 & 0 & 0 & 0 & 0 & 0 & 0 & 0 \\
\hline E29 & 0 & 0 & 0 & 0 & 0 & 0 & 0 & 0 & 0 & 0 & 0 \\
\hline E30 & 0 & 1 & 0 & 0 & 0 & 1 & 0 & 0 & 0 & 1 & 0 \\
\hline E31 & 0 & 0 & 0 & 0 & 0 & 0 & 0 & 0 & 0 & 0 & 0 \\
\hline E32 & 0 & 0 & 0 & 0 & 0 & 0 & 0 & 0 & 0 & 0 & 0 \\
\hline E33 & 0 & 0 & 0 & 0 & 0 & 0 & 0 & 0 & 0 & 0 & 0 \\
\hline E34 & 0 & 0 & 0 & 0 & 0 & 0 & 0 & 0 & 0 & 0 & 0 \\
\hline Total de errores & 2 & 2 & 3 & 0 & 2 & 3 & 1 & 1 & 4 & 4 & 0 \\
\hline
\end{tabular}

Fuente: Elaboración propia.

Las categorías que se analizaron en esta actividad se pueden ver en la tabla 10.

Tabla 10. Categorías y subcategorías actividad 1 (A1).

\begin{tabular}{|c|c|}
\hline Categoría & Subcategoría \\
\hline \multirow{6}{*}{$\begin{array}{c}\text { Categoría } \\
\text { 1. División y } \\
\text { conservación de } \\
\text { la unidad (grado) }\end{array}$} & Usa espacios diferentes al ubicar los números en el transportador. \\
\hline & No reconoce la unidad grado. \\
\hline & Secuencia numérica sin relación entre espacios y marcas. \\
\hline & No establece una secuencia numérica. \\
\hline & Importancia de las marcas y no de los espacios. \\
\hline & Ausencia de números en el transportador. \\
\hline \multirow{5}{*}{$\begin{array}{c}\text { Categoría } 2 . \\
\text { Ausencia del cero }\end{array}$} & Empieza la secuencia en 1. \\
\hline & Empieza la secuencia en 10. \\
\hline & Empieza la secuencia de números diferentes al cero y al 1 . \\
\hline & Empieza la secuencia de números diferentes al cero y al 10. \\
\hline & No ubica el cero en el transportador, pero deja su espacio. \\
\hline
\end{tabular}

Fuente: Elaboración propia.

Las respuestas de los estudiantes E6, E7 y E26 (figura 2), consideran la secuencia numérica en el transportador con números diferentes al 0 al 1 o al 10, en el caso de E7, inicia la secuencia en el ángulo de $90^{\circ}$, reconociendo correctamente el ángulo recto, pero finaliza con $440^{\circ}$ en el conteo, acierta en el reconocimiento de los ángulos de $180^{\circ}$ y $360^{\circ}$; uno de los casos poco frecuentes es el E18, quien realiza el conteo, no teniendo en cuenta la unidad grado del transportador, además de dejar la mitad inferior del transportador sin numeración, e iniciar el conteo desde 1, no reconoce el valor cero. Mientras que E17, E21 y E30 inician el conteo en 10 grados. 

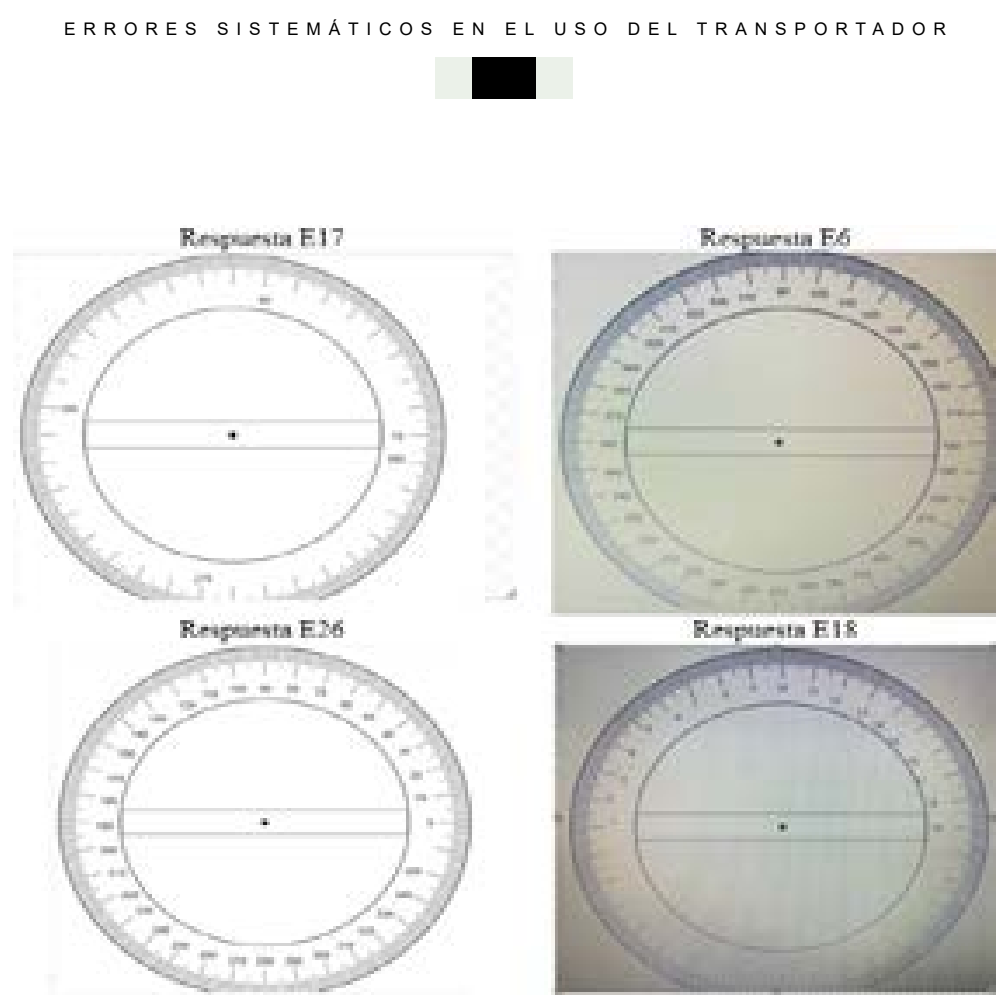

Figura 2. Respuestas estudiantes E17, E6, E26 y E18. Actividad 1 (A1). Elaboración propia.

\section{DISCUSIÓN ACTIVIDAD 1}

De los errores que surgen en la actividad 1 , se evidencian desde las categorías de análisis propuestas en magnitudes lineales, la adaptación realizada para el presente estudio permite identificar estos errores en el uso del transportador, por ejemplo, en las subcategorías donde se indica que el estudiante inicia los números en 1 o en 10, la actividad permitió reconocer los errores que al mismo tiempo fueron planteados por Levine et al. (2009) en su investigación sobre el aprendizaje de la medición de longitudes rectas, donde estudiantes de primaria y secundaria señalan que la regla comienza en 1 , y al contar marcas o números en una regla y no al reconocimiento de la unidad centímetro y su subdivisión milímetro.

A pesar de no ser un error frecuente en estudiantes de 13 a 15 años, debido a que han utilizado desde ciclo 2 el transportador para medir los ángulos es necesario resaltar que, no mencionar el número cero y además no dejar su espacio, incide en la relación número-medida que proponen Inhelder et al. (1974) donde manifiestan que los estudiantes hacen juicios de medición basados en ideas de conteo, en vez de iniciar con el número cero, debido a que asocian el número de conteo como una medida, en este caso, relacionan el número 1 o el número 10 con la medida de la amplitud angular.

En cuanto a la categoría conservación de la unidad, se reconoce que los estudiantes identifican inmediatamente una secuencia numérica, independientemente de sus escalas, el error radicó, en las marcas y los espacios, lo cual coincide con el señalamiento en magnitudes lineales de Macdonald (2011), en torno a, que los estudiantes no conservan la unidad, pero sí reconocen la secuencia numérica en el instrumento regla.

Los aportes que brindó los resultados de la actividad 1 , se enfocan a que el estudio de magnitudes lineales en torno a la medida $y$ el uso de la regla, permiten realizar aportes significativos para caracterizar y categorizar errores en el uso del instrumento transportador para la medida de la amplitud angular. Además, que permitió ampliar categorías de errores en el área de la geometría que ha sido poco aborda por los investigadores en didáctica de la matemática. 
Una propuesta para abordar y tratar el error de la ausencia del cero en el aula de clase, es diseñar actividades que permitan al estudiante visualizar el carácter dinámico del ángulo, por ejemplo, con movimientos del cuerpo donde la posición inicial indique el giro de cero grados; o también utilizar herramientas tecnológicas donde se puedan observar dos rayos sobrepuestos y se solicite girar uno de los rayos sobre el mismo vértice; el recorrido de un faro de ubicación de los barcos o aéreo, etc. Así, a partir de diferentes situaciones y contextos aplicados a situaciones reales, se puede aportar al estudiante herramientas donde sea necesario incluir el cero, al proceso de medición. Es allí donde radica la importancia de no definir el ángulo solamente desde la geometría euclidiana como se indicó en el marco referencial, sino también como región del espacio y como cantidad de giro.

\section{ACTIVIDAD 6 (A6)}

Observa el dibujo de la rueda de la fortuna y revisa los cuatro ángulos que se están señalando.

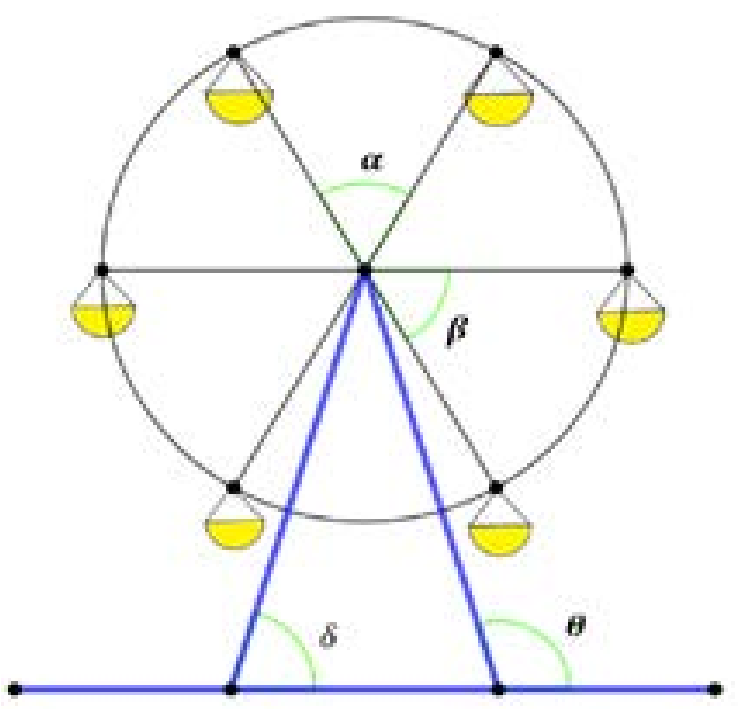

Figura 3. Rueda de la fortuna. Elaboración propia.

Usando el transportador, responde las siguientes preguntas:

¿Cuánto mide?

¿Cuánto mide?

¿Cuánto mide?

¿Cuánto mide? 
Tabla 11. Información recolectada y clasificada en categorías actividad 2 (A6).

\begin{tabular}{|c|c|c|c|c|c|c|c|c|c|c|c|c|}
\hline \multirow[t]{2}{*}{ Estudiante } & \multicolumn{12}{|c|}{ Categoría } \\
\hline & \multicolumn{4}{|l|}{3} & \multicolumn{3}{|c|}{4} & \multicolumn{5}{|c|}{6} \\
\hline E1 & 1 & 0 & 0 & 0 & 0 & 0 & 0 & 0 & 0 & 0 & 1 & 0 \\
\hline E2 & 0 & 0 & 0 & 0 & 0 & 0 & 0 & 0 & 0 & 0 & 0 & 1 \\
\hline E3 & 0 & 0 & 0 & 0 & 1 & 0 & 0 & 0 & 0 & 0 & 0 & 0 \\
\hline E4 & 0 & 0 & 0 & 0 & 0 & 1 & 0 & 0 & 0 & 0 & 0 & 0 \\
\hline E5 & 1 & 0 & 1 & 0 & 0 & 0 & 0 & 0 & 0 & 0 & 1 & 0 \\
\hline E6 & 0 & 0 & 0 & 0 & 0 & 0 & 1 & 0 & 0 & 0 & 1 & 0 \\
\hline E7 & 0 & 0 & 0 & 0 & 0 & 0 & 0 & 0 & 0 & 0 & 1 & 0 \\
\hline E8 & 0 & 0 & 0 & 1 & 0 & 0 & 0 & 0 & 0 & 0 & 0 & 0 \\
\hline E9 & 0 & 0 & 0 & 0 & 0 & 0 & 0 & 0 & 0 & 0 & 0 & 0 \\
\hline E10 & 0 & 0 & 0 & 0 & 1 & 0 & 0 & 1 & 0 & 1 & 0 & 0 \\
\hline E11 & 1 & 0 & 0 & 0 & 0 & 0 & 0 & 0 & 0 & 0 & 0 & 0 \\
\hline E12 & 0 & 0 & 0 & 0 & 0 & 0 & 0 & 0 & 0 & 0 & 1 & 0 \\
\hline E13 & 0 & 0 & 0 & 0 & 1 & 0 & 0 & 0 & 0 & 0 & 0 & 0 \\
\hline E14 & 0 & 0 & 0 & 0 & 0 & 0 & 0 & 0 & 0 & 0 & 0 & 0 \\
\hline E15 & 0 & 0 & 0 & 0 & 0 & 0 & 0 & 0 & 0 & 0 & 1 & 0 \\
\hline E16 & 0 & 0 & 0 & 0 & 1 & 0 & 0 & 0 & 0 & 0 & 0 & 0 \\
\hline E17 & 0 & 0 & 0 & 0 & 0 & 0 & 0 & 0 & 0 & 0 & 0 & 0 \\
\hline E18 & 0 & 0 & 1 & 0 & 0 & 0 & 0 & 0 & 0 & 1 & 0 & 0 \\
\hline E19 & 0 & 0 & 0 & 0 & 0 & 1 & 0 & 0 & 1 & 0 & 0 & 0 \\
\hline E20 & 0 & 0 & 0 & 0 & 0 & 0 & 0 & 0 & 0 & 0 & 0 & 0 \\
\hline E21 & 0 & 0 & 0 & 1 & 0 & 0 & 0 & 0 & 0 & 0 & 0 & 0 \\
\hline E22 & 0 & 0 & 0 & 0 & 0 & 0 & 0 & 0 & 0 & 0 & 0 & 1 \\
\hline E23 & 1 & 0 & 0 & 0 & 0 & 0 & 0 & 0 & 0 & 0 & 0 & 0 \\
\hline E24 & 1 & 0 & 0 & 0 & 1 & 0 & 0 & 1 & 0 & 0 & 0 & 0 \\
\hline E25 & 0 & 0 & 1 & 0 & 0 & 0 & 0 & 0 & 0 & 0 & 0 & 0 \\
\hline E26 & 0 & 0 & 0 & 0 & 0 & 0 & 0 & 0 & 0 & 0 & 0 & 0 \\
\hline E27 & 1 & 0 & 0 & 0 & 0 & 1 & 0 & 0 & 0 & 0 & 1 & 0 \\
\hline E28 & 0 & 0 & 0 & 0 & 0 & 0 & 0 & 0 & 0 & 0 & 0 & 1 \\
\hline E29 & 1 & 0 & 0 & 0 & 1 & 0 & 0 & 0 & 0 & 0 & 1 & 0 \\
\hline E30 & 1 & 0 & 0 & 0 & 0 & 0 & 0 & 0 & 1 & 0 & 1 & 0 \\
\hline E31 & 0 & 0 & 0 & 0 & 0 & 0 & 0 & 0 & 0 & 0 & 0 & 0 \\
\hline E32 & 0 & 0 & 1 & 0 & 0 & 0 & 0 & 0 & 0 & 0 & 0 & 1 \\
\hline E33 & 0 & 0 & 0 & 0 & 1 & 0 & 0 & 0 & 1 & 0 & 0 & 0 \\
\hline E34 & 0 & 0 & 0 & 0 & 0 & 0 & 0 & 0 & 0 & 0 & 0 & 0 \\
\hline Total de errores & 8 & 0 & 4 & 2 & 7 & 3 & 1 & 2 & 3 & 2 & 9 & 4 \\
\hline
\end{tabular}

Fuente: Elaboración propia. 
Las categorías que se analizaron en esta actividad son las siguientes

Tabla 12. Categorías y subcategorías actividad 6 (A6).

\begin{tabular}{|c|c|}
\hline Categoría & Subcategoría \\
\hline \multirow{3}{*}{$\begin{array}{l}\text { Categoría } 3 \text { Iteración } \\
\text { de la unidad grado y } \\
\text { aditividad }\end{array}$} & No suma espacios faltantes a las medidas \\
\hline & No resta espacios sobrantes a las medidas \\
\hline & $\begin{array}{l}\text { Cuenta los espacios, iterando la unidad hasta completar la medida de la } \\
\text { amplitud angular, sin considerar el punto final. }\end{array}$ \\
\hline \multirow{5}{*}{$\begin{array}{l}\text { Categoría 4. Relación } \\
\text { entre número y } \\
\text { medición }\end{array}$} & $\begin{array}{l}\text { Inicia el conteo desde el número } 1 \text { o el número } 10 \text {, donde se indica la } \\
\text { marca inicial del ángulo }\end{array}$ \\
\hline & $\begin{array}{l}\text { Asignar el número de la marca inicial y final en el transportador para } \\
\text { indicar la amplitud del ángulo. }\end{array}$ \\
\hline & Número de la marca como medida, en lugar de la amplitud angular. \\
\hline & $\begin{array}{l}\text { Asigna una unidad de medida diferente a la unidad de medida de la } \\
\text { amplitud angular (centímetro, metro, etc.). }\end{array}$ \\
\hline & No asigna unidad de medida. \\
\hline \multirow[t]{6}{*}{$\begin{array}{l}\text { Categoría } 6 \text {. Uso del } \\
\text { instrumento }\end{array}$} & $\begin{array}{l}\text { Considera el perímetro de la circunferencia como una regla para medir } \\
\text { la amplitud de los ángulos, no tiene en cuenta el vértice del ángulo para } \\
\text { realizar su medida. }\end{array}$ \\
\hline & $\begin{array}{l}\text { Considera el perímetro de la circunferencia como una regla para medir la } \\
\text { amplitud de los ángulos, ubica el vértice en un punto con referencia al cero } \\
\text { en el transportador. }\end{array}$ \\
\hline & $\begin{array}{l}\text { Mide la amplitud del ángulo, utilizando una regla de extremo a extremo a } \\
\text { los segmentos que conforman el ángulo. }\end{array}$ \\
\hline & $\begin{array}{l}\text { Mide la amplitud del ángulo, utilizando una regla para medir uno de los } \\
\text { segmentos que conforman el ángulo. }\end{array}$ \\
\hline & $\begin{array}{l}\text { No considera el centro del transportador para realizar la medición del } \\
\text { ángulo. }\end{array}$ \\
\hline & $\begin{array}{l}\text { No comprende la graduación interna y externa del transportador para } \\
\text { medir amplitudes. }\end{array}$ \\
\hline
\end{tabular}

Fuente: elaboración propia.

Para obtener la medida del ángulo, se obtuvo un total de 8 respuestas erróneas, los estudiantes $\mathrm{E} 1, \mathrm{E} 5, \mathrm{E} 27, \mathrm{E} 29$ y E30 miden el ángulo como se muestra en la figura 4 , donde ubican el vértice en la parte superior de la mirilla y realiza el conteo, encontrando que la medida es $55^{\circ}$, para los estudiantes E11, E23 y E24 consideran que la medida del ángulo es $65^{\circ}$, debido a que realizan el conteo iniciando en $10^{\circ}$. 


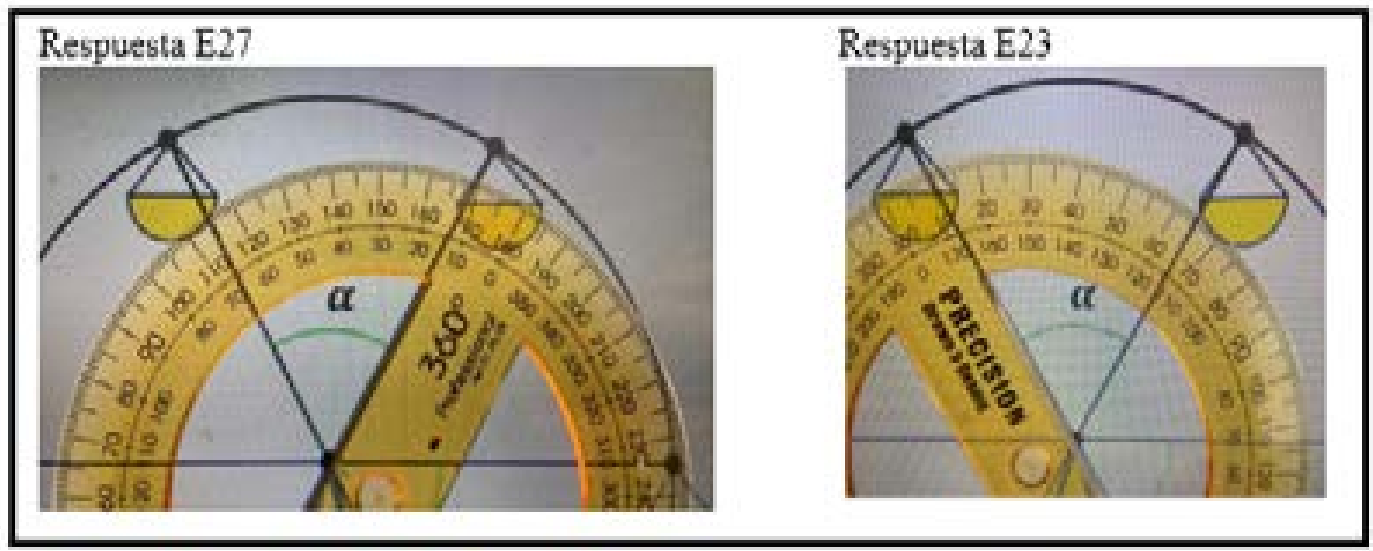

Figura 4. Respuestas de E27 y E23. A6. Elaboración propia

Para obtener la medida del ángulo, se encontró que el $41,17 \%$ de los estudiantes midieron incorrectamente el ángulo interno de un polígono, los estudiantes E5, E12 y E15 consideran que la medida del ángulo es $100^{\circ}$ debido que ubican el centro del transportador debajo de la mirilla y gradúan el segmento con el número cero (figura 4), los estudiantes E1, E6, E7 y E29 responden " $80^{\circ}$ ", este grupo de estudiantes ubica el vértice del transportador en la parte inferior de la mirilla de la circunferencia, y gradúan el rayo con cero grados (figura 4).

\section{DISCUSIÓN DE LA ACTIVIDAD 6}

Respecto a la medida de los ángulos y, los estudiantes cometen errores evaluados en las subcategorías de las actividades anteriores como la medida de segmentos para obtener la amplitud angular, no considerar la graduación interna o externa del transportador o la subdivisión de la unidad, la ausencia del cero, entre otros, es decir, se generó evidencia que al usar el instrumento físico en cada uno de los ángulos para medir la amplitud, recurrían a errores ya citados en la aplicación de la actividad.

Lo que indica, que la propuesta con el dibujo del transportador y de acuerdo con el objetivo específico "evaluar información mediante el diseño de una propuesta didáctica que permita recolectar los errores que cometen los estudiantes al usar el transportador de $360^{\circ}$ como instrumento de medida de la magnitud amplitud angular" permitió alcanzar el objetivo y además abordar la problemática expuesta en la investigación, a la luz de los autores que señalan errores en el área de la geometría y específicamente en la medición de la amplitud angular, del investigador, y de los resultados.

\section{DISCUSIÓN GENERAL}

En el análisis de resultados y en las discusiones 315 presentadas anteriormente se logró observar que el uso del transportador no es una tarea espontanea para los estudiantes, por lo cual, se requiere de producir información útil en aspectos de la planificación como la gestión del aula, la atención a la diversidad, la evaluación a los estudiantes, las tareas de los estudiantes para poder abordar y tratar el error. Es así, que la búsqueda de estrategias metodológicas en actividades de enseñanza aprendizaje no solamente se dan en torno al concepto de ángulo y la amplitud angular, también es importante para consolidar un conocimiento nuevo, prestar atención al uso del instrumento.

No obstante, el uso del instrumento debe permitir diferenciar al estudiante el objeto geométrico ángulo, las partes que lo componen y diferenciarlo de la amplitud angular. Aspecto que en muchas ocasiones los maestros pasan por alto, debido a que las actividades de clase o talleres que se proponen hacen referencia 
a "medir el ángulo" y esta configuración de palabras puede ser una de las causas que atraen al estudiante a medir no necesariamente la amplitud angular y en vez de ello, medir sus segmentos, su vértice, la posición del vértice con respecto a un punto de referencia en el transportador o la distancia entre los segmentos que componen el ángulo.

El instrumento diseñado permitió verificar los supuestos que se plantearon de acuerdo con los objetivos de la investigación y el planteamiento del problema, con respecto al supuesto del objetivo 1, Supuesto 1: Los errores sistemáticos se dan cuando se cometen con alta frecuencia en relación con las ocasiones que existen de tenerlos. Sisman y Aksu (2016) y Frías et al. (2001), establece algunos errores comunes y los conceptos erróneos en las tareas conceptualmente orientadas a procesos de medición; aunque lo hace en relación con la medición de longitud con el instrumento regla.

Tabla 13. Errores con el uso de la regla vs errores con el uso del transportador. Supuesto 1.

\begin{tabular}{|c|c|}
\hline Errores con el uso de la regla (E.R.) & \begin{tabular}{|c} 
Errores con el uso del transportador a partir de \\
investigaciones realizadas en el uso de la regla \\
(E.T.)
\end{tabular} \\
\hline $\begin{array}{l}\text { 1. E.R. Comprensión inadecuada de la } \\
\text { iteración, la aditividad y la estructura de una } \\
\text { regla. }\end{array}$ & $\begin{array}{l}\text { 1. E.T. No considerar el vértice del ángulo como } \\
\text { el punto del centro del transportador. Inadecuada } \\
\text { comprensión de la estructura del transportador. Y } \\
\text { no iterar adecuadamente la unidad al realizar el } \\
\text { conteo de la medida. }\end{array}$ \\
\hline 2. E.R. Iniciar de 1 en lugar de 0. & $\begin{array}{l}\text { 2. E.T. Iniciar el conteo de la medida angular en } 10 \\
\text { o en } 1 \text { en lugar de cero. }\end{array}$ \\
\hline $\begin{array}{l}\text { 3. E.R. Si una regla comienza con un número } \\
\text { distinto de cero, no se puede usar para medir. }\end{array}$ & $\begin{array}{l}\text { 3. E.T. Si uno de los lados del ángulo no marca el } \\
\text { cero, no es posible establecer correctamente la } \\
\text { medida del ángulo. Inadecuada comprensión del } \\
\text { punto } 0 \text {, iteración. }\end{array}$ \\
\hline $\begin{array}{l}\text { 4. E.R. Al medir, a partir de un determinado } \\
\text { punto (valor de la escala) de la regla la } \\
\text { longitud de un segmento, no restarle al } \\
\text { extremo el punto origen. }\end{array}$ & $\begin{array}{l}\text { 4. E.T. Al medir, a partir de un determinado punto } \\
\text { (valor de la escala) del transportador la amplitud } \\
\text { de un ángulo, no restarle al extremo el punto } \\
\text { origen. }\end{array}$ \\
\hline $\begin{array}{l}\text { 5. E.R. El alineamiento del extremo del } \\
\text { segmento que se ha de medir con el extremo } \\
\text { de la regla y no con el cero. Cuando el cero no } \\
\text { está en el extremo, puede inducir a errores en } \\
\text { las medidas. }\end{array}$ & $\begin{array}{l}\text { 5. E.T. Si el transportador no tiene el número cero } \\
\text { y en cambio tiene } 360^{\circ} \text { o } 180^{\circ} \text { la medición de la } \\
\text { amplitud angular es incorrecta }\end{array}$ \\
\hline $\begin{array}{l}\text { 6. E.R. Al medir un segmento, contar el } \\
\text { número de marcas correspondientes como } \\
\text { cantidad de unidades del segmento a medir. El } \\
\text { resultado es, entonces, una unidad mayor que } \\
\text { la medida real. }\end{array}$ & $\begin{array}{l}\text { 6. E.T. Al medir la amplitud angular, contar el } \\
\text { número de marcas correspondientes como } \\
\text { cantidad de unidades de la amplitud a medir. El } \\
\text { resultado es, entonces, una unidad mayor que la } \\
\text { medida real. }\end{array}$ \\
\hline
\end{tabular}

Fuente: elaboración propia. 
Efectivamente, cada uno de los errores consultados en el uso de la regla se lograron evidenciar en los resultados de la aplicación del instrumento, estos son: la comprensión del estudiante del concepto amplitud angular, las estrategias metodológicas en los procesos de enseñanza aprendizaje y la herramienta utilizada; esta última, en muchas ocasiones no es analizada por el docente, probablemente debido a la variedad de instrumentos que se encuentran en el mercado, sin embargo, es indispensable en todo proceso verificar el recurso didáctico que se está implementando, pues este, en sí mismo puede conducir a errores. Como se mostró en el desarrollo de las actividades, los estudiantes se dejan guiar por algunas marcas que parecen ser indispensables en el instrumento como la ubicación de la mirilla y no observan la concordancia de esta marca con la graduación cero del transportador. Aclaro, que no es que los instrumentos se encuentren mal diseñados, la reflexión se dirige en torno a algunos elementos extra que se demarcan en el transportador que pueden inducir a errores.

\section{CONCLUSIONES}

El análisis realizado de las respuestas dadas por los alumnos a distintos ítems de la actividad permitió extraer además de una discusión de resultados ya expuesta, una serie de conclusiones de carácter más global que dan respuesta a los objetivos planteados en la investigación y se exponen a continuación:

En algunas ocasiones consideran que la ausencia del cero no afecta la medición de la amplitud angular, es decir, el número no es indispensable en la medición de una amplitud y se les dificulta la conservación de la unidad, pero sí reconocen la secuencia numérica en el transportador, estudiantes en edades de 13 a 15 años le dan importancia a las marcas y a los espacios, en el reconocimiento del instrumento resaltan dos aspectos importantes, la secuencia numérica debe ser de 10 en 10 y los espacios en las marcas deben ser iguales en la ubicación de los números, pero si se cambia la escala implica un cambio de unidad y algunos afirman que ya no es posible medir amplitudes angulares.

Respecto a las marcas que señalan la unidad en el transportador, en ocasiones cuentan las marcas en vez de los espacios, es decir logran iterar la unidad de la amplitud angular, pero establecen una medida incorrecta debido a la concepción que tienen de la medición.

Otro error asociado al reconocimiento de la unidad (1), está relacionado con la medida de la amplitud angular, debido a que los estudiantes aproximan a unidades que sean múltiplos de 10 , es decir, en ocasiones no reconocen las subdivisiones de cada $10^{\circ}$ en la cual se encuentra graduado el transportador y además este error está asociado con la continuidad de una magnitud.

Existen investigaciones alrededor de la conservación de la magnitud, los cuales hacen énfasis que el cambio de ubicación, forma o tamaño su magnitud permanece constante, sin embargo, no hay estudios específicos en torno a medir con un transportador de diferente tamaño, al respecto, los estudiantes consideran que un transportador al ser más grande, las marcas se encontraran a mayor distancia o que el tamaño del instrumento afecta la medida, ejemplo, con un transportador más grande se obtienen amplitudes más grandes de un mismo ángulo.

La lectura del transportador es más sencilla cuando utilizan la parte superior del instrumento, con la parte inferior muestran una mayor dificultad, los estudiantes no se encuentran familiarizados con los ángulos de $180^{\circ}$ a $360^{\circ}$ y con la lectura de esta parte del transportador. 


\section{REFERENCIAS BIBLIOGRÁFICAS}

Abrate, R., Pochulu, M., y Vargas, J. (2006). Errores y dificultades en matemáticas. Análisis de causas y sugerencias de trabajo. Provincia de Córdoba, Argentina: Universidad Nacional de Villa María. Obtenido de http://unvm.galeon. com/Libro1.pdf

Astolfi, J. (1999). El "error", un medio para enseñar. Colección Investigación y Enseñanza, núm. 15. Sevilla: Diada.

Brousseau, G., Davis, R., y Werner, T. (1986). Observing Students at Work. In: Christiansen B., Howson A.G., Otte M. (eds) Perspectives on Mathematics Education. Mathematics Education Library, vol 2. Springer, Dordrecht. https://doi.org/10.1007/978-94-0094504-3 6

Cantoral, R., Farfán, R., Cordero, F., Alanís, J., Rodríguez, R., y Garza, A. (2005). Desarrollo del Pensamiento Matemático. Ciudad de México, México: Trillas.

Casas, L., y Luengo, R. (2005). Conceptos nucleares en la construcción del concepto de ángulo. Enseñanza de las Ciencias, 23(2), 201 - 216.

Chamorro, M. (1995). Aproximación a la medida de magnitudes en la enseñanza primaria. En Uno. Revista de didáctica de las matemáticas, 3, 31-53.

Franchi, L., y Hernández, A. (2004). Tipología de errores en el área de la geometría plana parte II. La revista Venezolana de Educación, 8(25), 196-204.

Frías, A., Gil, F. y Moreno, M. F. (2001). Introducción a las magnitudes y la medida. Longitud, masa, amplitud y tiempo. En E. Castro (Ed.), Didáctica de la Matemática en la Educación Primaria (pp. 477-502). Madrid, España: Síntesis.

Guzmán, A. (2014). Propuesta didáctica para la enseñanza de ángulos y su medida a estudiantes de grado séptimo a partir de la recreación de algunos instrumentos de posicionamiento astronómico (tesis de maestría). Universidad Nacional de Colombia Bogotá D.C, Colombia. Recuperado de http://bdigital.unal.edu. co/12925/1/2806902.2014.pdf

Inhelder, B., Sinclair, H., y Bovet, M. (1974). Apprentissage et structures de la connaissance. [Learning and the structures of cognition]. Presses Universitaires de France.

Levine, S.C., Kwon, M. K., Huttenlocher, J., Ratliff, K., y Dietz, K. (2009). Children's understanding of ruler measurement and units of measure: A training study. In Proceedings of the Annual Meeting of the Cognitive Science Society, 31 (31), 2391-2395.

Macdonald, A. (2011). Young children's representations of their developing measurement understandings. Mathematics: Traditions and [new] practices, 1, 482-490.

Ministerio de Educación Nacional, (2003). Estándares básicos de competencias en matemáticas. Bogotá. Obtenido de https://www.mineducacion.gov.co/1621/ articles-116042_archivo_pdf2.pdf

Mitchelmore, M., y White, P. (1998). Development of Angle Concepts: A framework for research. Mathematics Education Research Journal, 10(3), 4 - 27. doi: 10.1023/A:1003927811079

https://sci-hub.tw/https://link.springer. com/article/10.1007/BF03217055 
Movshovitz, H., Zaslavsky, O., y Inbar S. (1987). An empirical classification model for errors in high school mathematics. Journal for research in mathematics education, 18 (1), 3-14. doi: $10.2307 / 749532$

Pochulu, M. (2005). Análisis y categorización de errores en el aprendizaje de la matemática en alumnos que ingresan a la universidad. Revista Iberoamericana de Educación, 4, 1-15.

Rico, L. (1995). Errores y dificultades en el aprendizaje de las matemáticas. En J. Kilpatrick, P.

Gómez, y L. Rico (eds.), Educación Matemática, 69-96. Bogotá: Grupo Editorial Iberoamérica.

Rotaeche, R., y Montiel, G. (2017). Aprendizaje del concepto escolar de ángulo en estudiantes mexicanos de nivel secundaria. Educación matemática, 29(1), 171 - 199. doi: 10.24844/ EM2901.07

Rotaeche, R., y Montiel, G. (2009). La construcción del concepto de ángulo en estudiantes de secundaria. Aportaciones para un diseño escolar. Acta Latinoamericana de Matemática Educativa, Vol. 22. México, DF: Colegio Mexicano de Matemática Educativa A. C. y Comité Latinoamericano de Matemática Educativa.

Sisman, G., y Aksu, M. (2016). A study on sixth grade students' misconceptions and errors in spatial measurement: Length, Area, and Volume. International Journal of Science and Mathematics Education, 14(7), 1293-1319. doi:10.1007/s10763015-9642-5
Socas, M. (1997). Dificultades, obstáculos $y$ errores en el aprendizaje de las matemáticas en la educación secundaria. En L. Rico (Coord.) La educación matemática en la enseñanza secundaria (pp. 125-154). Barcelona: Horsori.

Vasilachis, I. (2006). Estrategias de Investigación Cualitativa. Barcelona: Gedisa. 